\title{
Research Paper \\ Comparison of Two Approaches for Measuring Moral Development: Neo-Kohlbergian Approach and Moral Study Challenges
}

\author{
Zahra Hooshyari ${ }^{1},{ }^{*}$ Ali Delavar² ${ }^{2}$ Asghar Minaei ${ }^{3}$, Hossein Eskandari $^{4}$
}

1. PhD in Measurement and Assessment, Department of Evaluation and Measurement, Faculty of Psychology and Educational Sciences, Allameh Tabataba'i University Tehran, Iran.

2. PhD in Measurement and Assessment, Full Professor, Department of Evaluation and Measurement, Faculty of Psychology and Educational Sciences, Allameh Tabataba' University, Tehran, Iran.

3. PhD in Methodology and Statistics, Assistant Professor, Department of Evaluation and Measurement, Faculty of Psychology and Educational Sciences, Allameh Tabataba'i University, Tehran, Iran.

4. PhD in Psychology, Associate Professor, Department of Psychology, Faculty of Psychology and Educational Sciences, Allameh Tabataba'i University, Tehran, Iran.

\begin{tabular}{|c|c|}
\hline $\begin{array}{l}\text { Use vour devicict to san } \\
\text { and read the article online }\end{array}$ & dttation: Hooshyari Z, Delavar A, Minaei A, Eskandari H. [Comparison of Two Approaches for Measuring Moral \\
\hline 口斯的回 & Development: Neo-Kohlbergian Approach and Moral Study Challenges (Persian)]. Iranian Journal of Psychiatry and Clinical \\
\hline Atsuts & Psychology. 2018; 24(1):30-43. https://doi.org/10.29252/NIRP.IJPCP.24.1.30 \\
\hline 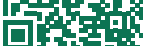 & dol": https://doi.org/10.29252/NIRP.IJPCP.24.1.30 \\
\hline
\end{tabular}

Received: 19 Aug. 2017

Accepted: 01 Nov. 2017

Key words:

Defining Issue

Test, Sociomoral

Reflection Measure-

Short Form, Moral

judgment inter-

view, Hypothetical

dilemmas, In-depth

interview

\section{A B S TR A C T}

Objectives A few of the challenges involved in measuring moral development includes tools and methodology. The present study compared two neo-Kohlbergian approaches in the study of moral development.

Methods A total of 40 peoples referred to the Center for Behavioral and Mental Health Assessment were selected as a sample group, subjected to QUAN-QUAL (equal) design, and assessed using Defining Issue Test (DIT), Sociomoral Reflection Measure-Short Form (SRM-SF), semi-structured interviews based on hypothetical moral dilemmas, and in-depth interviews based on real-life dilemmas.

Results The results showed that DIT and SRM-SF scores were correlated in the moral maturity index; however, these two methods and the results of conflicts in real life exhibited significant differences . Conclusion Therefore, standards and instructions were applied to both methods including test based on the memory or recalling that would yield similar results. Furthermore, the difference between these two methods with respect to results and interviews based on real-life dilemmas might be dependent on the situation of the individual than the tools, making self-judgment easier for individuals.

\section{Extended Abstract}

\section{Introduction}

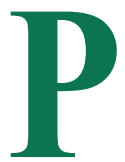

sychological constructs have various implications that may or may not be appropriate for measurement [1], and hence, morality is extremely controversial [2]. Although Kohlberg changed the morality into a measurable subject [6], it yet faces several challenges. Kohl- berg demonstrated subjects with nine dilemmas and want them to make decision for story hero and explain how to obtain the solution. The subjects' reasoning is criteria for their moral development $[11,12,14]$. Herein, it is presumed that a person is aware of his mind's inner processes and can express them [15]. Despite the establishment of the validity and reliability of Kohlberg method, it was severely criticized [12, 14, 17], Due to the lack of evidence for the post-conventional stage [16], this method exhibits limitations in self-reporting of cognitive processes and access to (n.......

* Corresponding Author:

Ali Delavar, PhD

Address: Department of Evaluation and Measurement, Faculty of Psychology and Educational Sciences, Allameh Tabataba'i University, Tehran, Iran Tel: +98 (912) 1300129

E-mail: delavarali@yahoo.com 
underlying information [18]. Since the mental operation is elusive from cognitive assessment $[19,20]$ and since the interview depends on the conscious understanding, people are unaware of the content of their minds $[15,18]$. Thus, the two Neo-Kohlbergian approaches, led by James Rest and John Gibbs, overcame these criticisms.

Moreover, people know more than they say and weakness in verbal ability should not limit them from expressing their reasoning. They may not remember their knowledge, but if encountered with the same reasoning, they can identify and score them $[16,19,26]$. Thus, after the participants encounter dilemma, instead of putting forth their reasoning, they receive a questionnaire that includes the reasoning item that should be rated for the importance of its role in dilemma solving. The participants give a high score to items that match to their dominant moral schema unconsciously. The moral development level of the participant is defined by the maximum scores for reasoning $[8,16,19$, 20, 21]. Gibbs did not encounter people with a dilemma, rather asked them to explain how much and why some of the ethics such as devotion, honesty, affection, and justice were a necessity. The individual's answers formed the basis for the determination of the stage of moral development, and Gibbs expressed the reasons stated by the subjects according to Kohlberg [23].

The remaining participants presented evidence for postconventional moral stage and eliminated the impact of verbal skills in moral development measured as a strong point of the approach. However, this method was criticized by one participant who did not make reasoning by their-self fragment [16]. For Gibbs, the cognitive maturation allowed the processing of information, analysis, and conclusion; if a person could not reason for a high stage, it indicated that the higher stage was not consensual [7, 12, 14, 17, 23]. However, if the two methods measured the unit construct, a consensus was essential [33]. Thus, the present study compared the two methods with each other and with the external criteria (interviews based on hypothetical riddles and real-life conflicts in individuals).

\section{Method}

The present study is based on the mixed method models [34]. The research sample comprised of 40 respondents to Behavioral and Mental Health Assessment Center in December 2016, who were selected through purposeful sampling. The tools used included Defining Issue Test (DIT), Sociomoral Reflection Measurement-Short Form (SRM$\mathrm{SF}$ ), moral interviews based on the hypothetical dilemma, and in-depth interviews based on real life. Data were ana- lyzed using Kendall's method, Pearson's correlation coefficient, analysis of variance, and independent t-test.

\section{Results}

The correlation between P of Defining Issue Test (DIT) and SRM is 0.62 , while that between subjects' moral development in SRM-SF and DIT is 0.54. All individuals that are labeled as morally mature persons by SRM-SF have a normative or post-conventional moral schema in DIT. However, of the 12 individuals that SRM-SF identified as morally immature, only 5 in the DIT received an interested personal schema. The participants in 3 moral schemas were compared via SRM scores, and the results showed the difference between PISs with NMSs and PCSs. The results also confirmed the difference between individuals with and without moral maturity (based on SRM) with respect to $\mathrm{P}$ derived from DIT. The correlations between the results of hypothesis-based interviews and the results of DIT and SRM-SF were significant and 0.46 and 0.62 , respectively. The correlations between the results of the in-depth interview based on personal real-life events and the results of DIT and SRM-SF were significant and 0.48 and 0.53 , respectively. Thus, a significant difference between DIT results and interview results of real-life events.

\section{Discussion}

The correlation coefficient observed between the two tools is an index of their coherence in unit construct measurement [43]. It was also observed that in SRM, PIS had a significantly lower score than NMS and PCS. Although the PIS person wanted the world according to his desire, an attitude towards partnership and adherence to rules and principles was lacking. On the other hand, NMS prefers adherence to rules, order, and equality in law for all due to the moral maturity and avoid tribalism and self-orientation. The PCS individuals wanted welfare beyond the rules for all humans and exhibited a high moral maturation in SRM score. The SRM identifications were also confirmed by DIT; those with moral maturation earned a $\mathrm{P}$ as an indicator of post-conventional thinking and cannot be observed those without moral maturation.

The correlation coefficient confirmed using two tools with external criteria that served as the best indicators of the reliability of the results. Moreover, we observed that all the participants in DIT with a high score in NMS or PCS did not necessarily display the same in real-life; this result was not observed for SRM-SF. There is no hero in SRM, and therefore, no third person, voice of judger, and subject in one. Also, in personal life, the story's hero and the one who is reasoning are the same individuals. Furthermore, the 
emotions [33, 44, 45], sense of duty [46], thought of the outcome, and the fate of the closest people [32] affects the individual's decision and maybe a moral act independent of moral judgment in reality [49]

\section{Ethical Considerations}

\section{Funding}

This paper was extracted from the first author's $\mathrm{PhD}$ dissertation in the Department of Evaluation and Measurement, Faculty of Psychology and Educational Sciences, Allameh Tabataba'i University of Tehran.

\section{Conflict of Interest}

The authors declare no conflict of interest. 


\title{
مقايسه دو رويكرد مبتنى بر يادآورى و بازشناسى در اندازهَّيرى رشد اخلاقى: نئوكلبركَىها و جالشهاى بررسى رشد اخلاقى
}

\author{
زهرا هوشيارى'، "على دلاورّ، اصغر مينايى"؛ حسين اسكندرى"
}

1- دكتراي سنجش و اندازهميرى، كروه سنجش و اندازهميرى، دانشكده روانشناسى و علوم تربيتى، دانشكاه علامه طباطبايى، تهران، ايران.

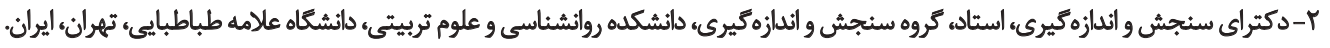

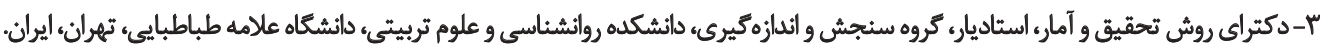

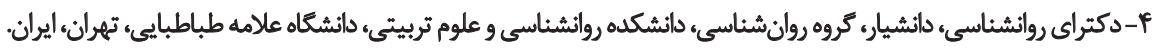

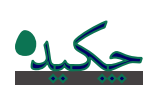

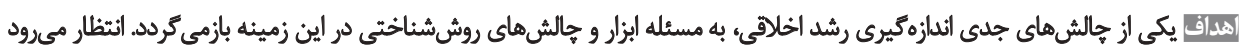

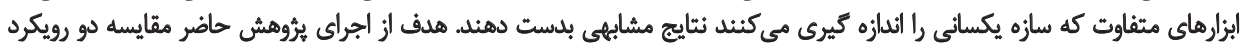
تئوكلبركى در بررسى رشد اخلاقيى استى رئ.

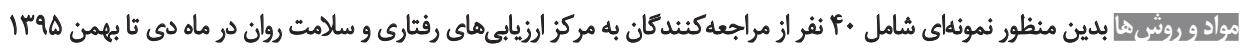

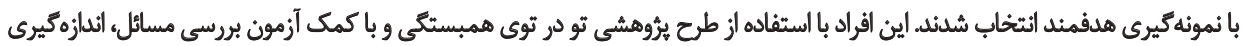

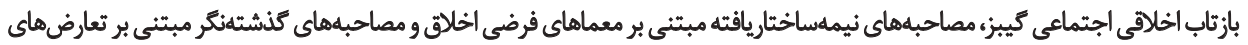
زندكى واقعى فرد آزموده شلديند.

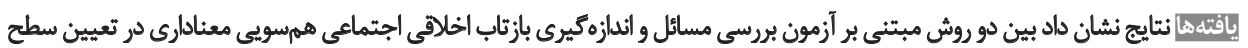

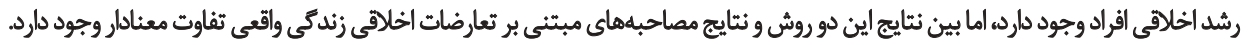

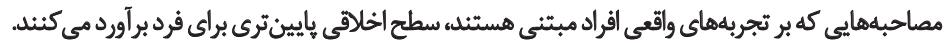

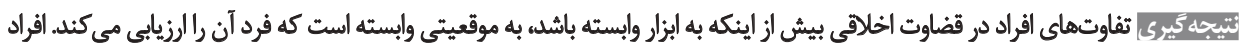

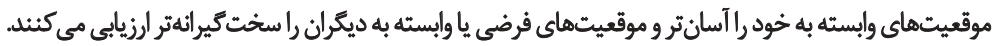

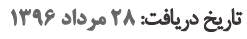

تاريخ بذيرش: 10

\section{كليدواثرها:}

آزمون بروسي مسائل،

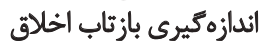

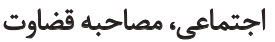

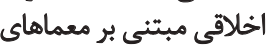
فرضي، مصاحبه عميق ميتي

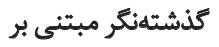

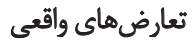

كردند [ع]. كلبرى با برداختن به رشد تحولى فهم افراد از عدالت

مقامه

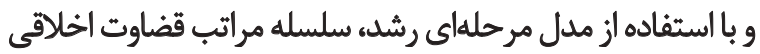

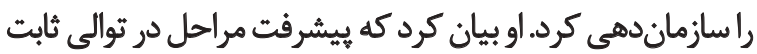

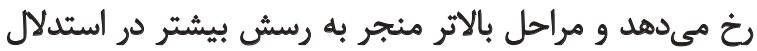

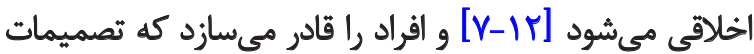

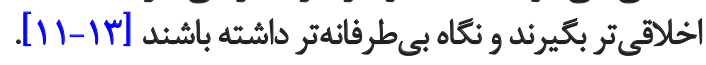
كلبرى آزمودنى ها را با معماهاى اخلاقي مواجهها كرد؛؛ راه حل

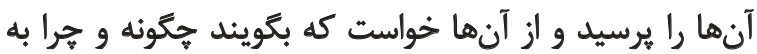

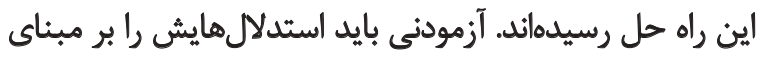

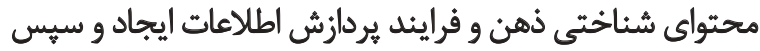

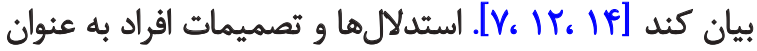

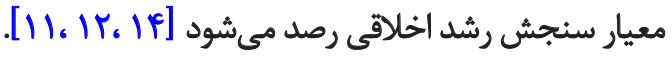

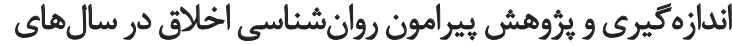

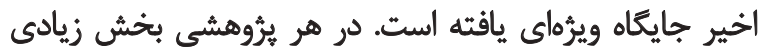

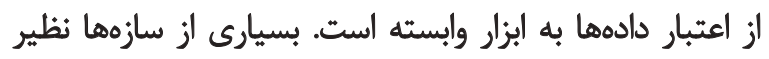

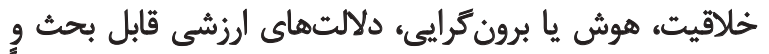

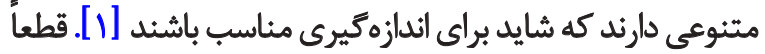

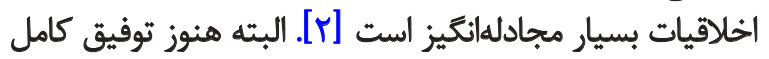

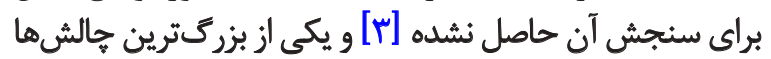

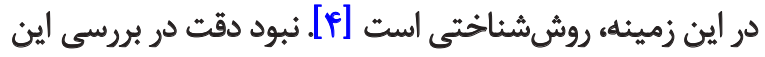

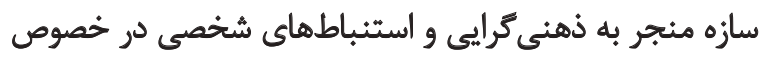

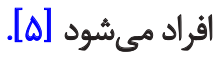

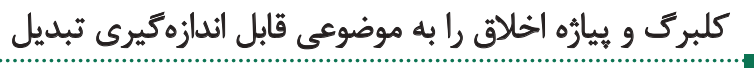
دكتر على دلاور نشانى: تهران، دانشعاه علامه طباطبايى، دانشكده روانشئاسى و علوم تربيتى، كروه سنجش و اندازمكيرى.

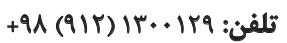

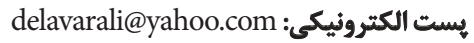


اقراد مي خواست توضيح دهند كه هرا فلان تصميم را كرفتهانده

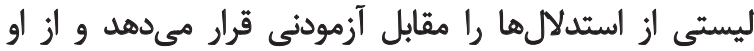

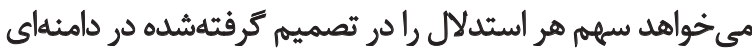

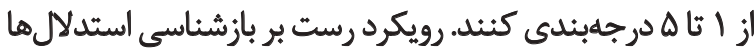

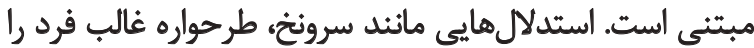

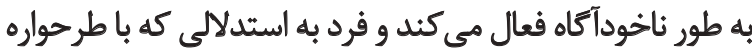

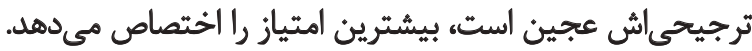

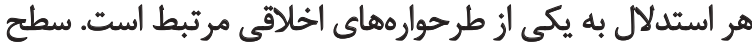

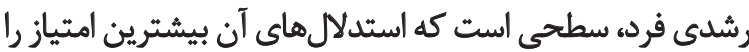

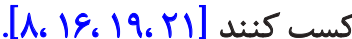

كيبز افراد را با معماهاى اخلاقى روبهرو نمى كند. او از افراد

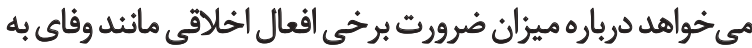

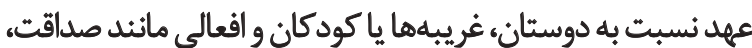

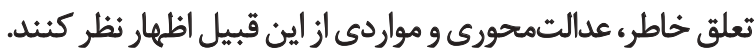

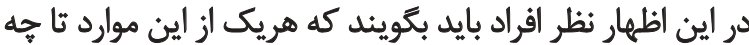

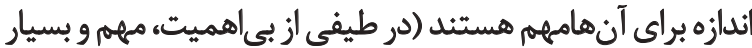

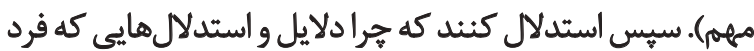

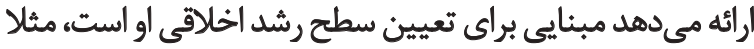

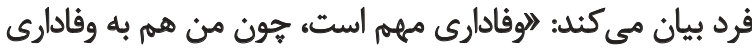

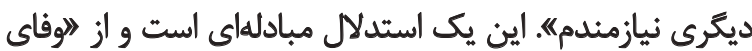

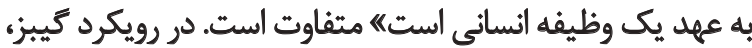

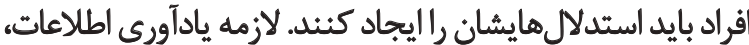

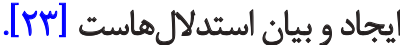

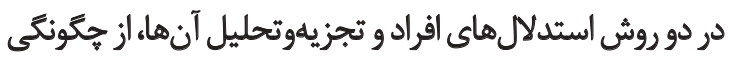
تصميم در موقعيتهاى اخلاقي، توجه به مئه ميزان مسئوليت و و

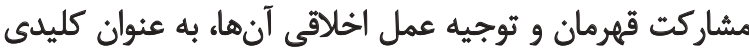

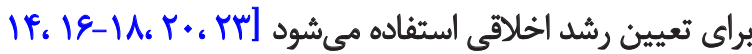

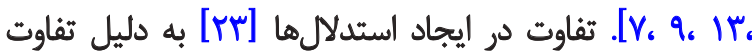

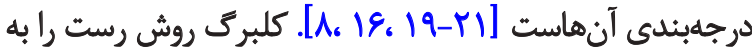

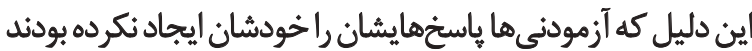

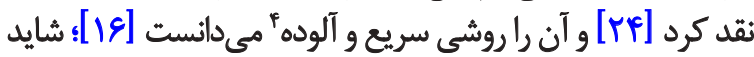

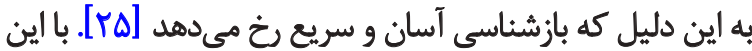

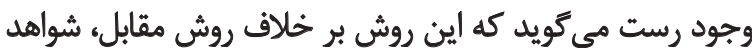

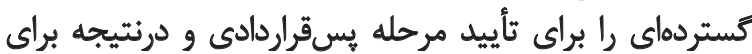

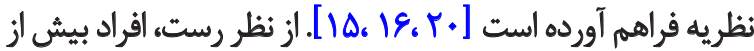

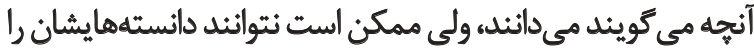

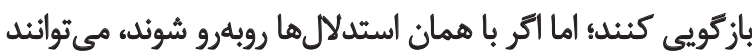

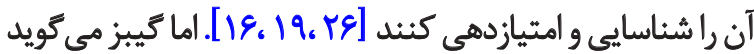

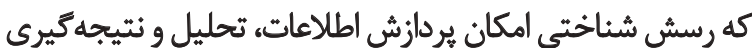

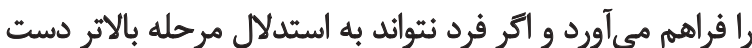

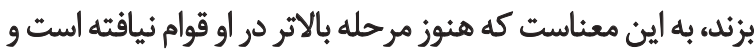

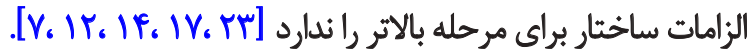

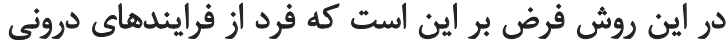

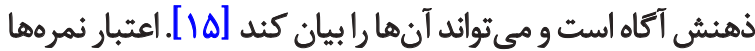

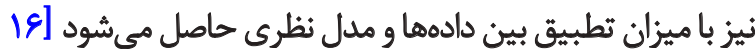

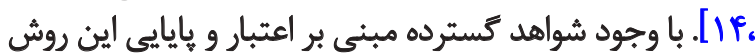

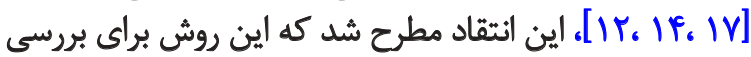

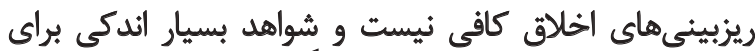

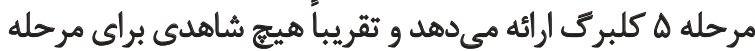

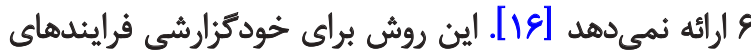

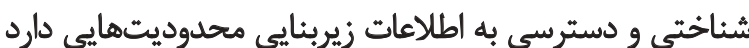

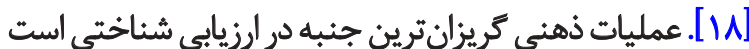

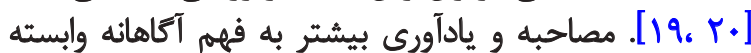

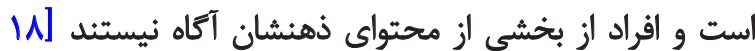

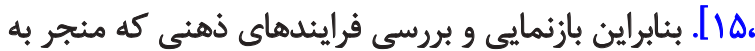

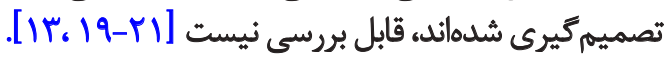
از اينرو دو رويكرد تئوكلبركى با رهبرى جيمز رست و جان

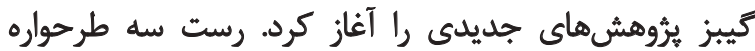

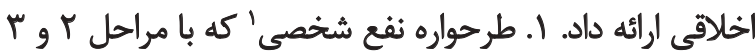

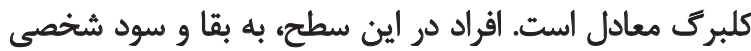

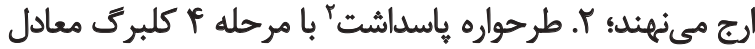

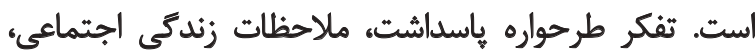

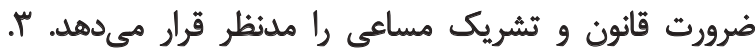

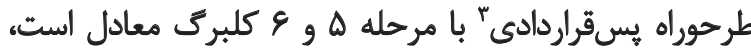

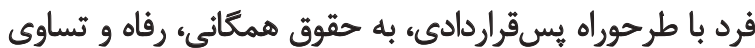

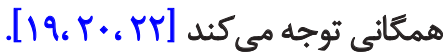
كيبز براى رشد اخلاقى F أمرحله در نظر كرفت و بر اساس

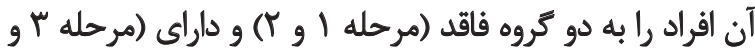

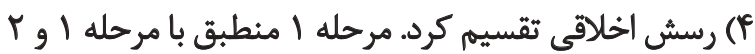

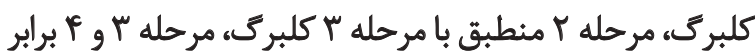

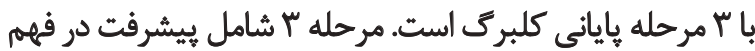

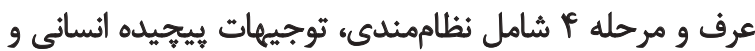

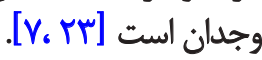

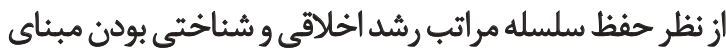

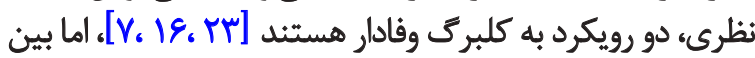

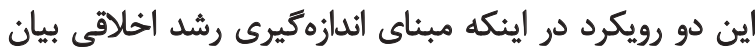

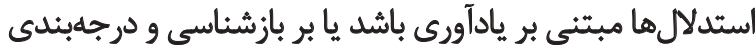

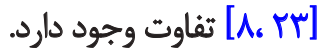
رست افراد را با معماهاى اخلاقى مواجهيه كرد. افراد بايد

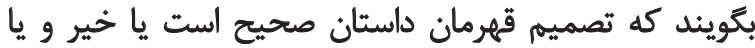
نمىتوانيد تصميم بكيرند. در ادامه، او بر خلاف كلبرى استى كه از

1. personal Interests Schema

2. Maintaining Norms Schema

3. Post conventional Schema 


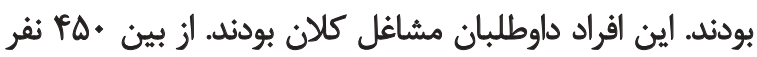

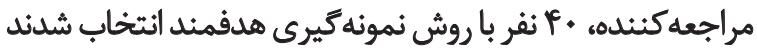

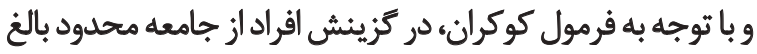

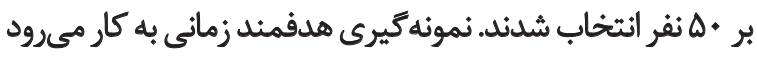

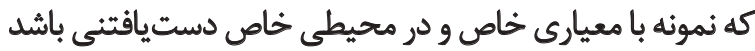

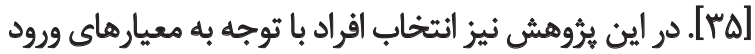

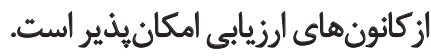

معيارهاى ورود عبارت است از: داشتن حداقل نمره توائمندى آنى

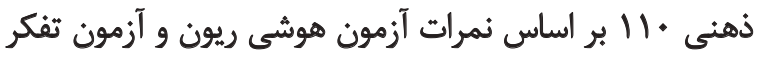

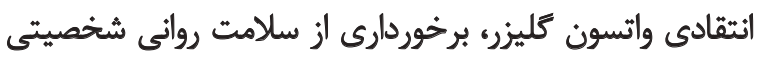

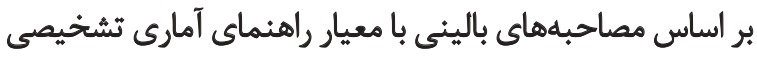

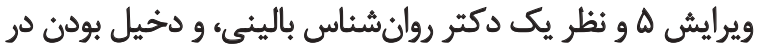

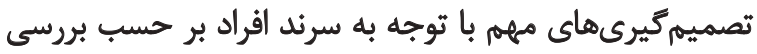

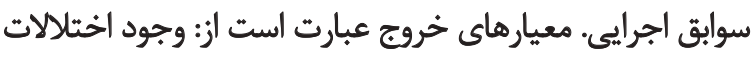

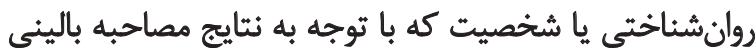

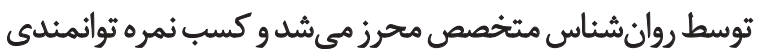

$$
\text { ذهنى كمتر از ·.11. }
$$

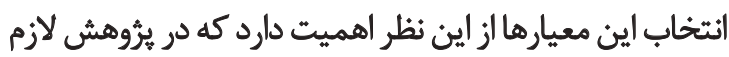

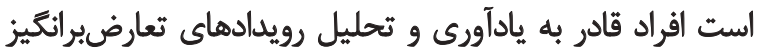

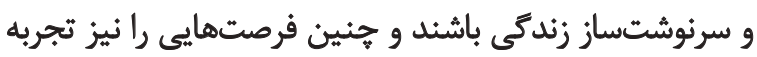

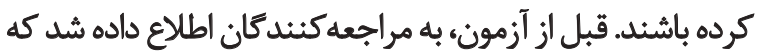

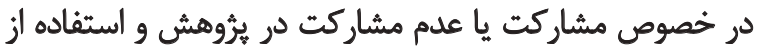

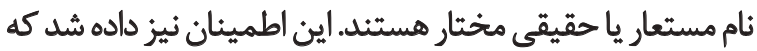

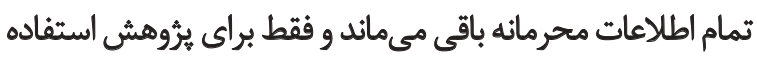

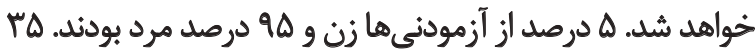

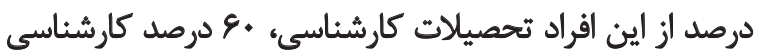

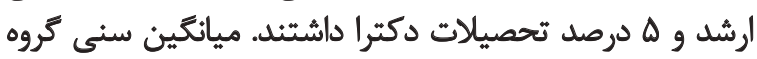

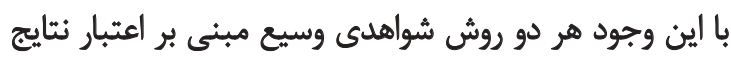

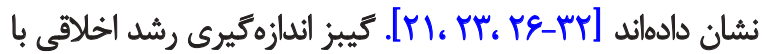

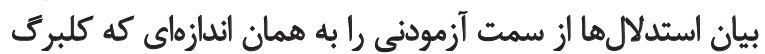

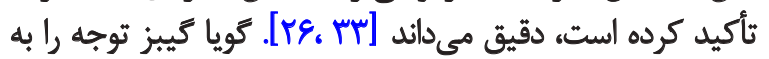

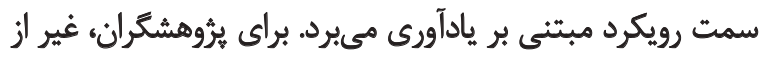

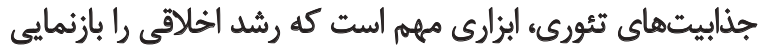

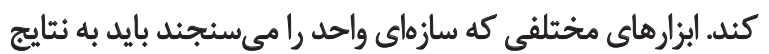

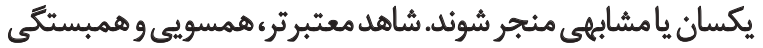

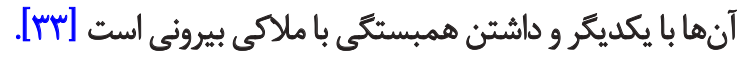
تاكنون بثوهشها هر ابزار را به طور جداكانه بررسى كردماند.

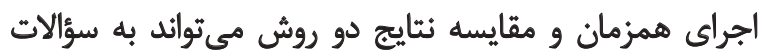

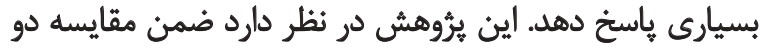

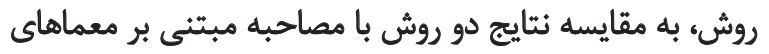

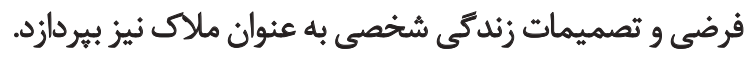

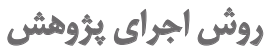

در اين بروهش طرح تو در توى مدل همبستكى از كروه

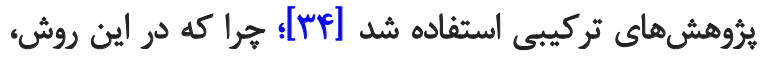

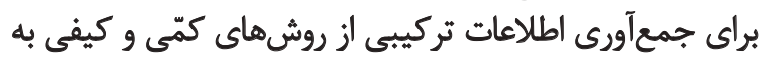

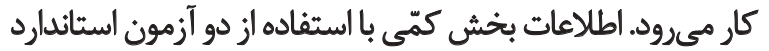

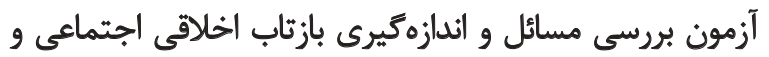

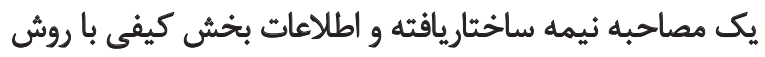
مصاحبه هاى عميق اكتشافى جمعآورى شد. جامعه يُوهش شامل مراجعه كنندكان به مركز ارزيابى رفتارى

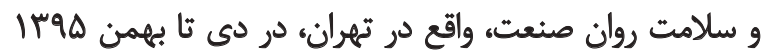

جدول ا. اطلاعات دموكرافيك كروه نمونه

\begin{tabular}{|c|c|c|}
\hline مشاهدهده (\%) & ابعاد & شاخص ها \\
\hline$r(\Delta)$ & زن & \multirow{2}{*}{ جنسيت } \\
\hline$r \wedge(9 \Delta)$ & هرد & \\
\hline$I F(r \Delta)$ & كارشئاسى & \multirow{3}{*}{ تحصيلات } \\
\hline$M f\left(e_{0}\right)$ & كارششئاسى ارشد & \\
\hline$r(\Delta)$ & دكترا & \\
\hline$M(A Y / \Delta)$ & هديريتى & \multirow{2}{*}{ شعل } \\
\hline$V(I V / \Delta)$ & سريرستى & \\
\hline$P(1)$. & كمتر از • P ب & \multirow{3}{*}{ نسن } \\
\hline$W(P Q)$ & Q. b. & \\
\hline$M(P Q)$ & هيشتر أز ه. & \\
\hline
\end{tabular}

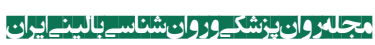


جدول Y. مقايسه نتايج دو آزمون اندازهكيرى بازتاب اخلاقى اجتماعى - فرم كوتاه و بررسى مسائل

\begin{tabular}{|c|c|c|c|c|c|c|c|c|c|}
\hline \multicolumn{4}{|c|}{ شاخصهاى آمارى } & \multicolumn{4}{|c|}{ اندازهيرى باز تاب اخلاقى اجتماعى - فرم كوتاه } & & \\
\hline \multirow[t]{2}{*}{ Sig. } & $\mathbf{r}$ & Sig. & تاي كندال & درك عرف & سيستم & خودكامكى & مباديلداي & & \\
\hline & $\begin{array}{l}\text { P and } \\
\text { SRM } \\
\text { Score }\end{array}$ & & سلسلهمراتب & خقى (تعداد) & رسش & فلاقى (تعداد) & ثارسشى 1 & & \\
\hline \multirow{4}{*}{.1 .1} & \multirow{4}{*}{. I8ro* } & \multirow{4}{*}{.1 .1} & \multirow{4}{*}{.$(\Delta)^{p e *}$} & \multicolumn{2}{|c|}{ - } & \multicolumn{2}{|l|}{$\Delta$} & نقع فردى & \\
\hline & & & & \multicolumn{2}{|c|}{ r. } & \multicolumn{2}{|l|}{$v$} & 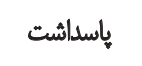 & 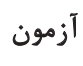 \\
\hline & & & & \multicolumn{2}{|c|}{$A$} & \multicolumn{2}{|l|}{ - } & 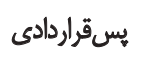 & مسائلى \\
\hline & & & & \multicolumn{2}{|c|}{ TA } & \multicolumn{2}{|c|}{ ir } & جمع & \\
\hline
\end{tabular}

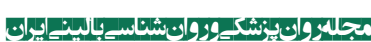

علامت *** به معناى اطمينان آمارى 99 • است.

جدول با. مقايسه نمرات اندازهيرى بازتاب اخلاقى اجتماعى در افرادى با طرحوارهاي آزمون بررسى مسائل

\begin{tabular}{|c|c|c|c|c|c|}
\hline مقايسههاي دوتايى & Sig. & $\mathbf{F}$ & درجات آزادى & 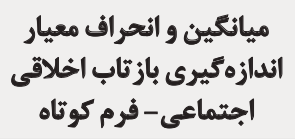 & \\
\hline PIS \& MSN* & $.1 \cdot 1$ & If/Arem & $m, r$ & $\operatorname{VEr}(\cdot / F \Delta)$ & تُفع فُردى \\
\hline PIS \& PCS* & & & & $\operatorname{T/PV}(\cdot / \Delta \rho)$ & ياسدائشت \\
\hline MSN \& PCS & & & & $m / M P(\cdot / M q)$ & يسقراردادي \\
\hline
\end{tabular}

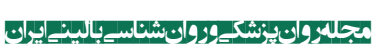

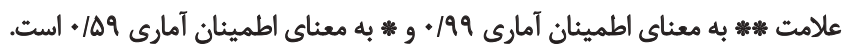

جدول F. مقايسه نمرات آزمون بررسى مسائل در افرادى با طبقهبندى اندازميرى بازتاب اخلاقى اجتماعى- فرم كوتاه

\begin{tabular}{|c|c|c|c|c|}
\hline Sig. & $\mathbf{t}$ & درجه آزادى & ميانتين Pو انحراف معيار & كروه بثدى \\
\hline \multirow[t]{2}{*}{$\% 1$} & r/gr"** & 山ᄉ & $r / / V(V / \cdot F)$ & فاقد رسش اخلاقى \\
\hline & & & หจ/ภ१(ร/१४) & داراى رسش اخلاقى \\
\hline
\end{tabular}

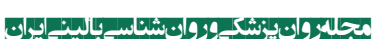

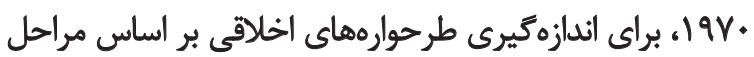

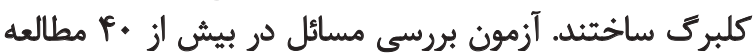

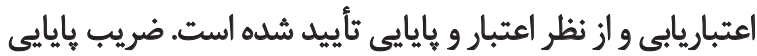

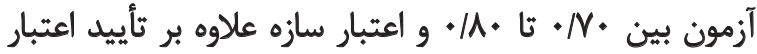

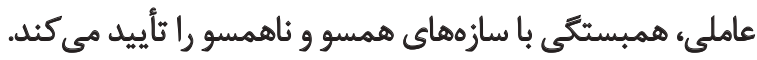

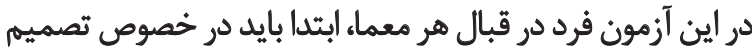

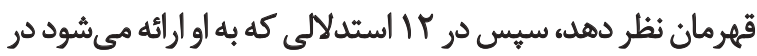

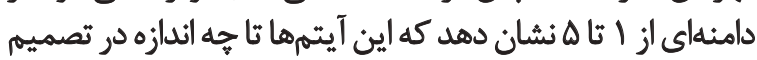

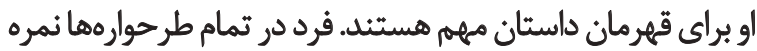

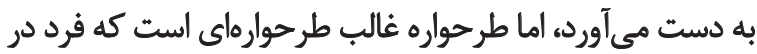

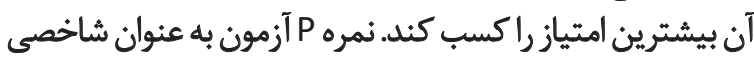

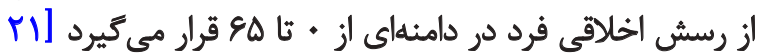

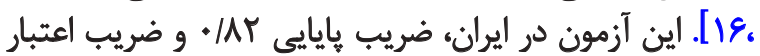

علامت *** به معناى اطمينان آمارى 99 • است.

مطالعه fV و انحراف معيار ه/IT بود. تمام شركت كنيندكان در

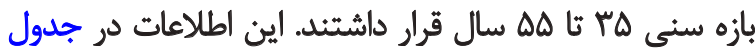

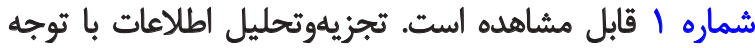

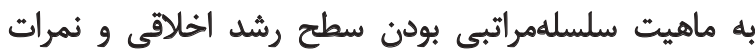

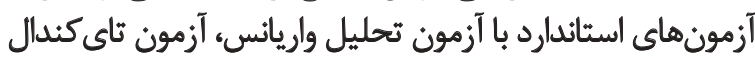

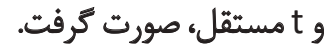

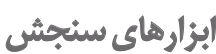

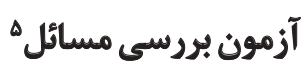

رست و همكاران اين آزمون را با نام اختصارى DIT در سال

5. Definition Issue Test 
جدول ه. هقايسه ئتايج آزمون بررسى مسائل و اندازهيرى بازتاب اخلاقى اجتماعى - فرم كوثاه با مصاحبههاي نيمه ساختاريافته

\begin{tabular}{|c|c|c|c|c|c|c|c|}
\hline \multirow{2}{*}{ Sig. } & \multirow{2}{*}{ ثاي كندال } & \multicolumn{4}{|c|}{ مصاحبههاي مبتنى بر معماهاي فرضي (تعداد) } & \multirow{2}{*}{ ملاك ابزارها } & \multirow{2}{*}{ آزمون } \\
\hline & & 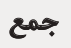 & 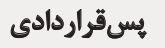 & باسداشت & نفع فردى & & \\
\hline \multirow{4}{*}{.1 .1} & \multirow{4}{*}{$+/ 48 * *$} & $\Delta$ & $\cdot$ & $r$ & $r$ & تفع فردى & \multirow{4}{*}{ آزمون بررسيى } \\
\hline & & rv & * & $\pi$ & r & 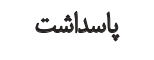 & \\
\hline & & $\wedge$ & 1 & $\checkmark$ & - & يس قراردادى & \\
\hline & & f. & 1 & r & 8 & 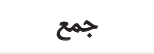 & \\
\hline \multirow{3}{*}{$.1 \cdot 1$} & \multirow{3}{*}{$.18 Y^{* * *}$} & ir & - & 8 & 8 & نارسى اخلاقى & \multirow{3}{*}{ اجتازتاب اخلاقى - اندازيرى } \\
\hline & & rA & 1 & rv & - & رسش اخلاقى & \\
\hline & & r. & 1 & $m$ & 8 & جمع & \\
\hline
\end{tabular}

مطالعه در ب كشور در خارج از ايالات متحده استفاده شده

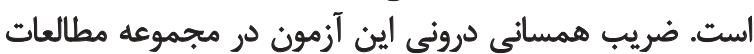

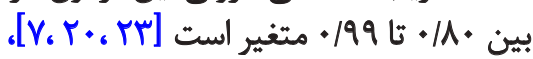

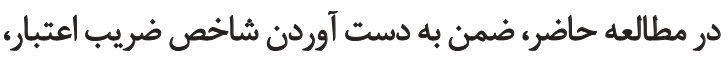

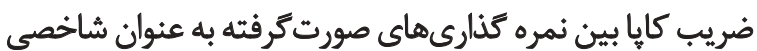

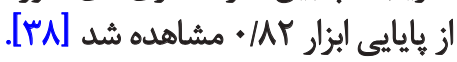

\section{مصاحبههاى مبتنى بر معماهاى فرضى}

مصاحبه هاى نيمه ساختاريافته متشكل از ه معما و هر معما

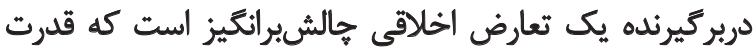

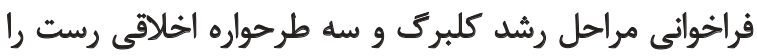

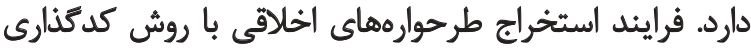

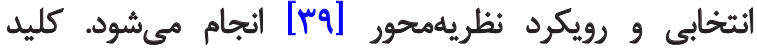

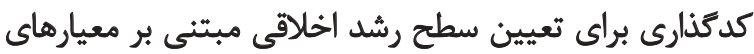

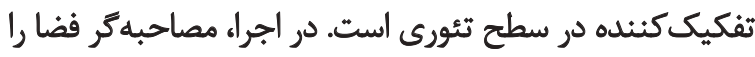
براى استخراج استدلال هاى حداكثرى آزمودنى بدون درن القاكنيدئى

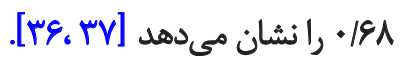

اندازهيرى باز تاب اخلاقى اجتماعى - فرم كوتاه' كيبز در سال بر19 إين برسشنامه را ساخت. در اين آزمون

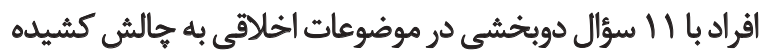

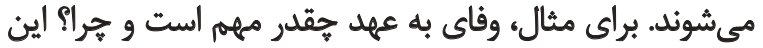

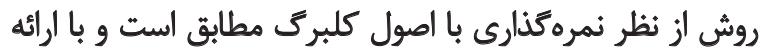

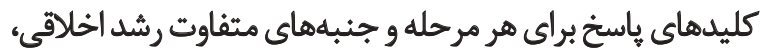

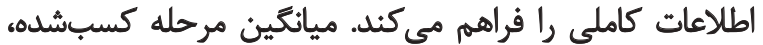

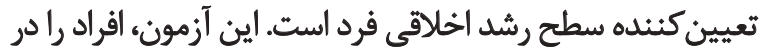

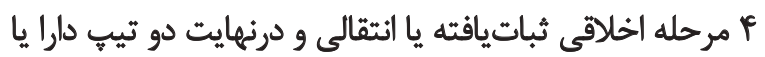

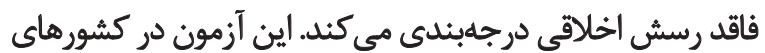

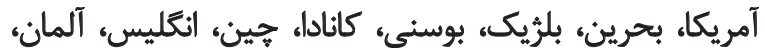

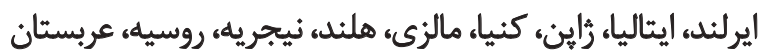

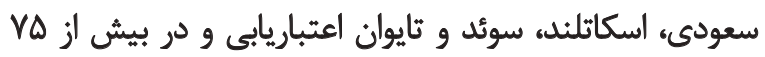

6. Socio-moral Reflection Measure- Short Form (SRM-SF)

جدول و. مقايسه نتايج آزمون بررسي مسائل و اندازهميرى بازتثاب اخلاقي اجتماعي با مصاحبههاي مبثنى بر تعارضات زندكى واقعى

\begin{tabular}{|c|c|c|c|c|c|c|c|}
\hline \multirow[t]{2}{*}{ Sig. } & \multirow{2}{*}{ ثناي كندال } & \multicolumn{4}{|c|}{ مصاحبههاي مبتنى بر رويدادهاى والقعى } & \multirow{2}{*}{ ملاك ابزارها } & \multirow{2}{*}{ آزمون } \\
\hline & & جمع & يسقراردادى & نياسداشت & نفع فردى & & \\
\hline \multirow{4}{*}{.1 .1} & \multirow{4}{*}{.$/ 4 A^{* *}$} & $\Delta$ & . & . & $\Delta$ & نفع فردى & \multirow{4}{*}{ آزمون بروبئي } \\
\hline & & IV & . & 18 & 11 & 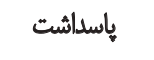 & \\
\hline & & $\wedge$ & 1 & 8 & 1 & 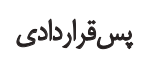 & \\
\hline & & r. & 1 & M & iv & 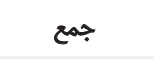 & \\
\hline \multirow{3}{*}{$.1+1$} & \multirow{3}{*}{$. / 01)^{2+*}$} & ir & - & r & 1. & 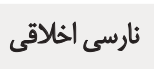 & \multirow{3}{*}{ بازتاب اخلازئى الجتمى } \\
\hline & & ra & 1 & r. & $\checkmark$ & رسش اخلاقى & \\
\hline & & r. & 1 & MT & IV & جمع & \\
\hline
\end{tabular}




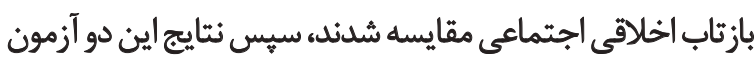
به ترتيب با مصاحبههاى نيمه ساختاريافته و و عميق مقايسه

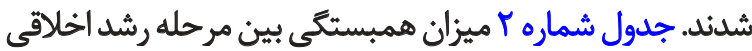

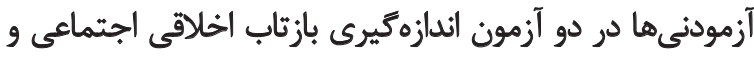

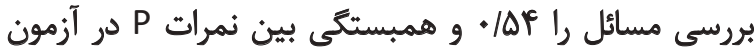

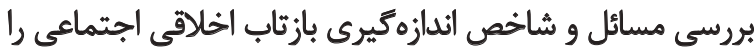

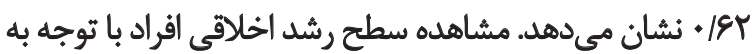

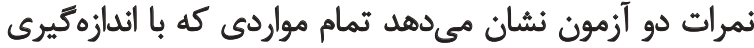

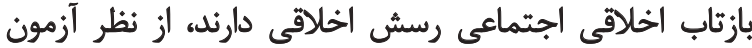

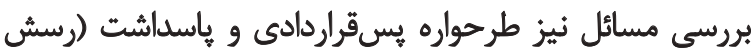

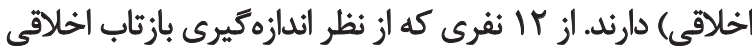

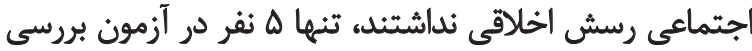

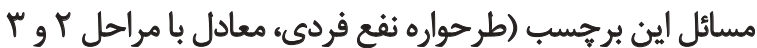
كلبرى و عدم رسش اخلاقى كيبز) را دريافت كردند. جدول شماره ז، افراد داراى ب طرحواره را از نظر نمرات

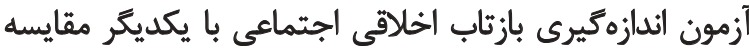

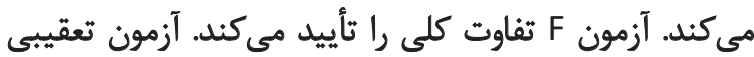

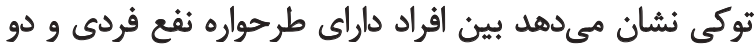

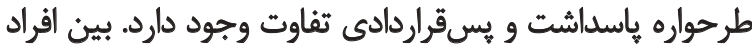

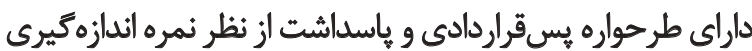

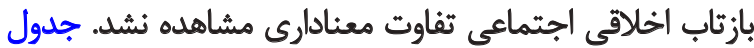

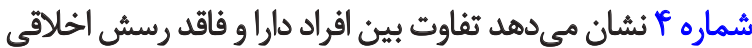

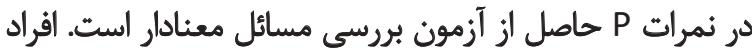

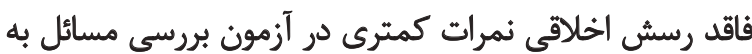

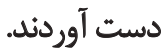

جدول شماره ه، ارتباط دو آزمون و مصاحبههاى مبتنى بر مدرب

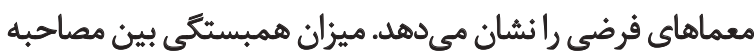

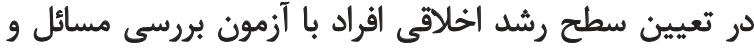

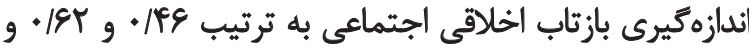
توزيع افراد در هر طبقه در همين جدول قابل جابل مشاهده است.

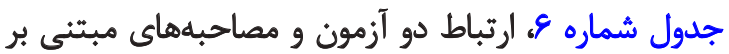

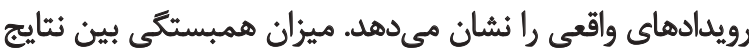

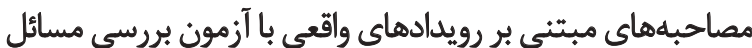

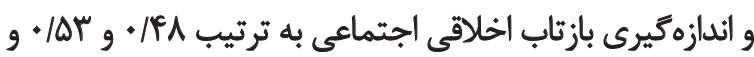
توزيع افراد در هر طبقه در اين جدول قابل إنافي مشاهده است ترني

بحث

نتايج نشان مى دهد دو آزمون نتايج يكديكر را تأييد مى كنيند.

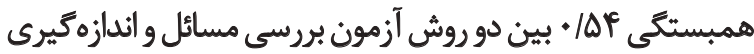

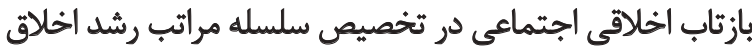

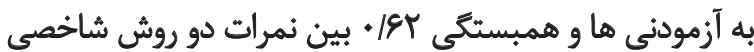

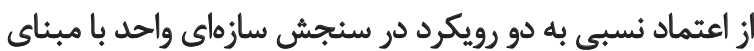
تئورى واحد است [بr]. شاهدى ديكر بر همسيك داريى دو دو ابزار

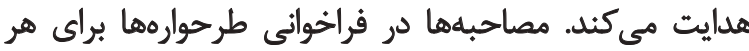

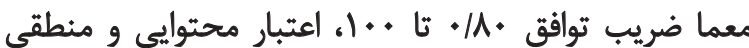

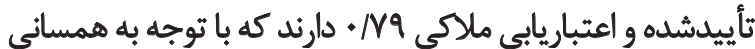

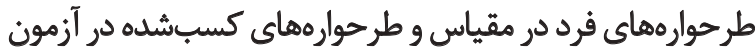

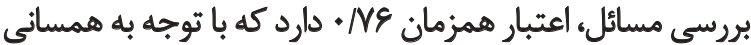

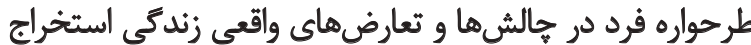

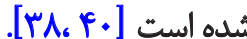

اعتبار ملاك بيشبين با توجه به همبستكى بين مصاحبه

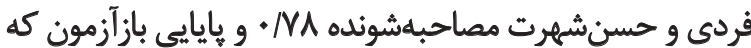

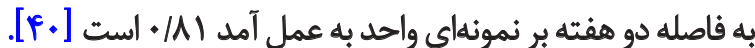

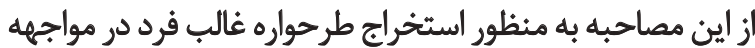

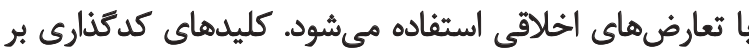

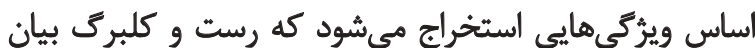

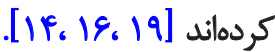

\section{مصاحبههاى مبتنى بر رويدادهاى زندتى واقعى}

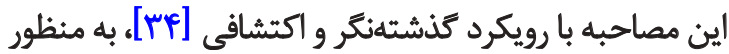

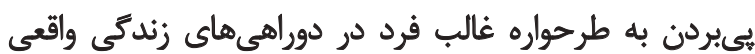

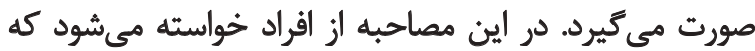
تعارضهاى مواجههشده در زندكى واقعى را بيان كنند و بكتويند

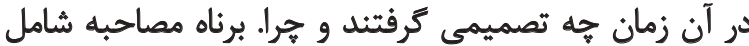

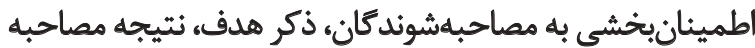

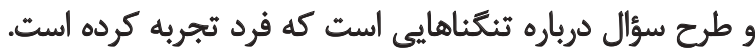

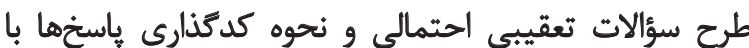

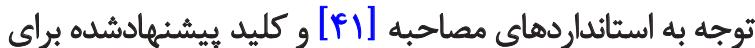

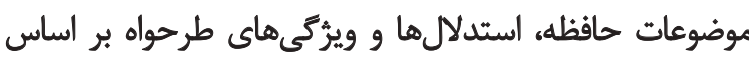
نظريه طرحوارههاى اخلاقى صورت بذيرفت [19، 19].

$$
\text { روش إجرا }
$$

ابتدا افراد مورد مصاحبه نيمه ساختاريافته قرار ترفت، سيس به به إزيه

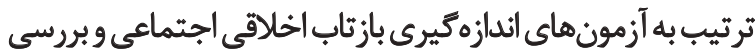

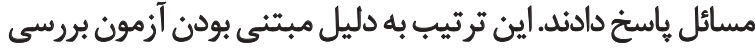

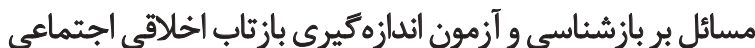

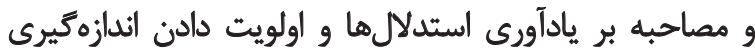

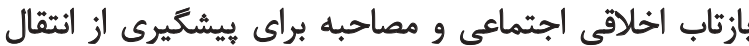

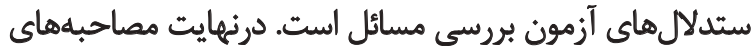

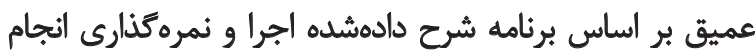

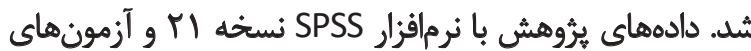

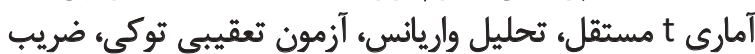
همبستكى بيرسون و تاي كندال تحليل شدند.

ياقتهها

در اين ثبروهش ابتدا دو آزمون بررسى مسائل و اندازهكيرى 
آزمون بررسى مسائل سطح رشد اخلاقى نشان داد تمام اقرادى

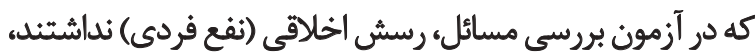

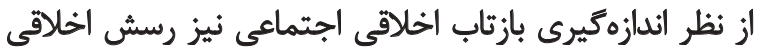

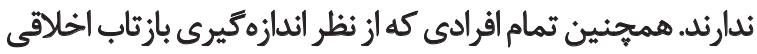

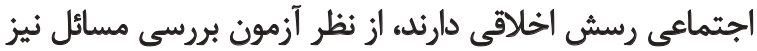

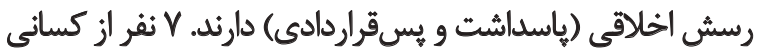

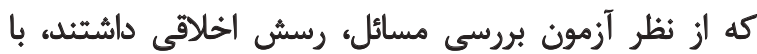

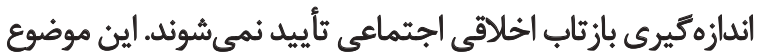

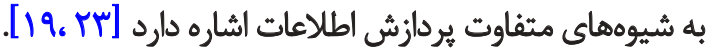

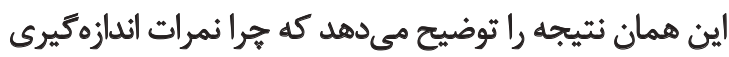

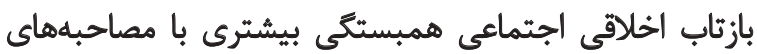

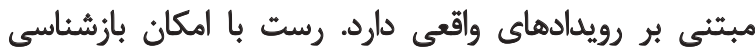

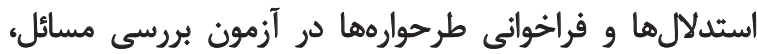

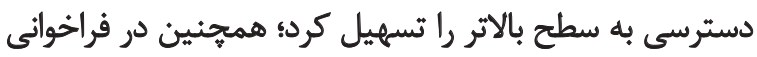

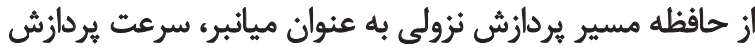

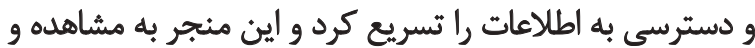

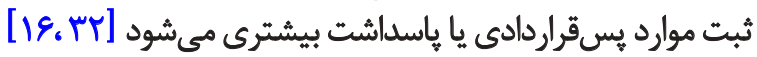

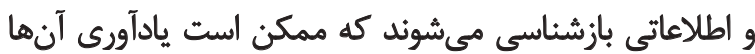

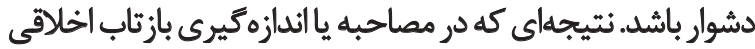

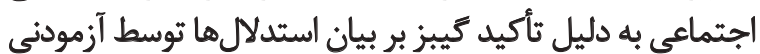

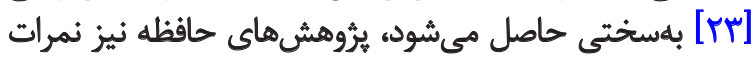

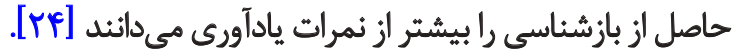

نتيجه قابل ملاحظه ديكر در همين راستا، مشاهده موارد

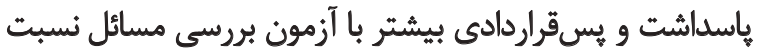

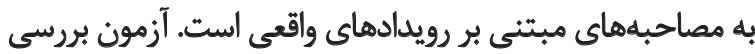

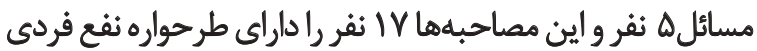

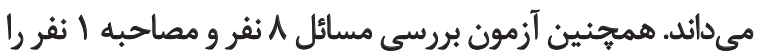

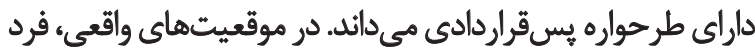

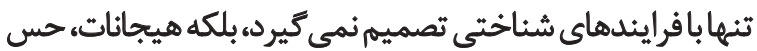

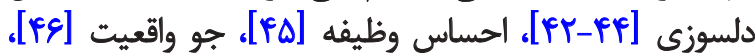

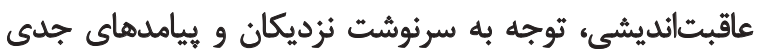

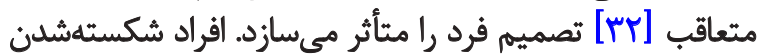

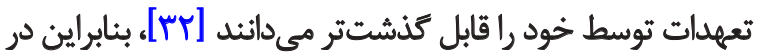

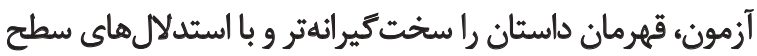

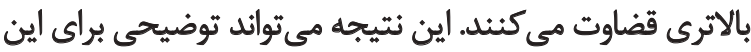

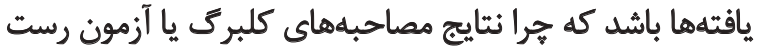

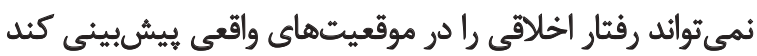

[FV, FA]

برخى يُوهشها نشان مى دهند عمل اخلاقى ممكن است از

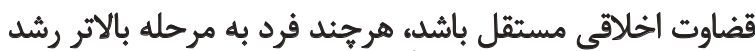

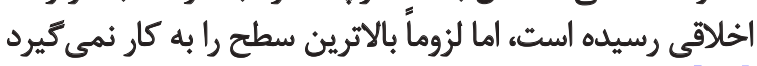

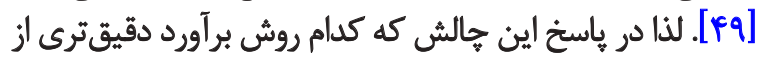

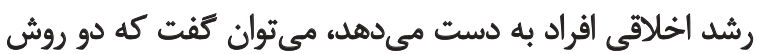

اين است اقراد داراى طرحواره نفع فردى نسبت به افراد داراي

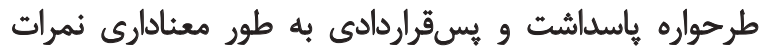
كمترى در اندازهيرى بازتاب اخلاقى اجتماعى كسب كردانداند.

افراد داراي طر حواره نفع فردى، جهان مناسب راجائي مي دانيند

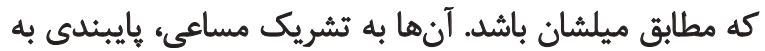

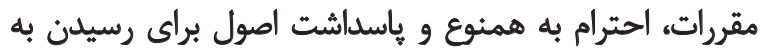

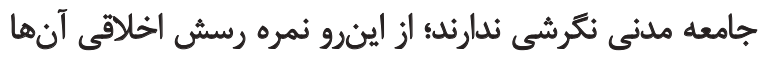

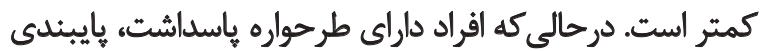

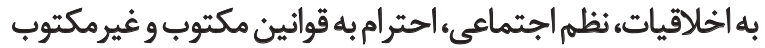

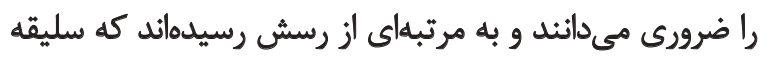

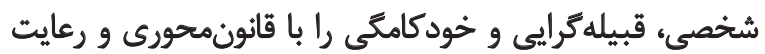

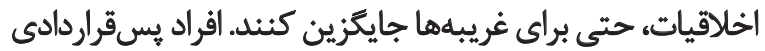

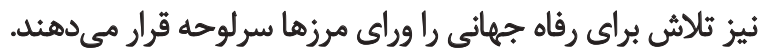

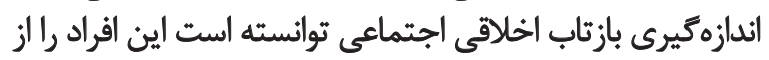

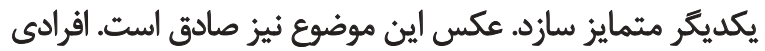

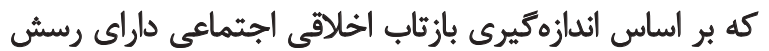

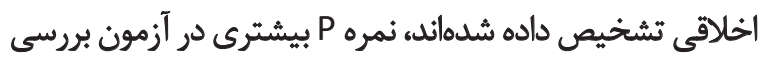

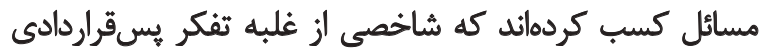

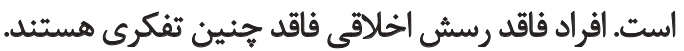
همبستكى نتايج آزمون بررسى مسائل و معيارهاى بيرونى

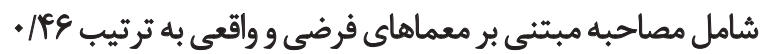

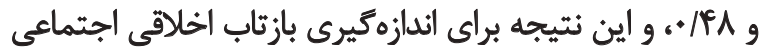

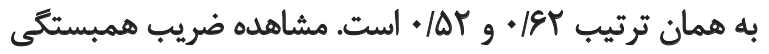

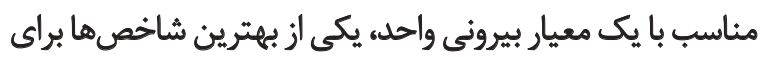
اعتماد به نتايج آزمونهاى مربوط بير به دو رويكرد است.

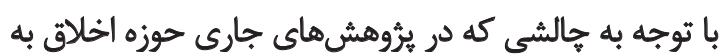

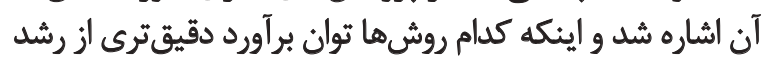

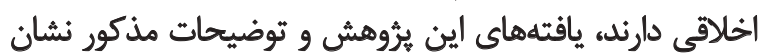

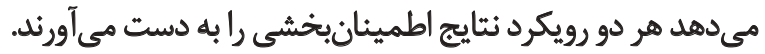

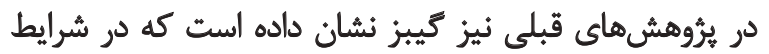

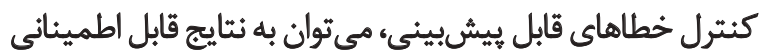

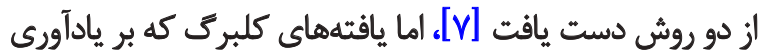

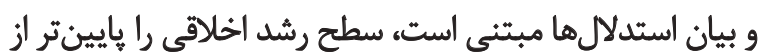

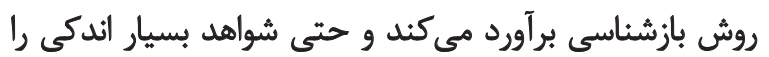

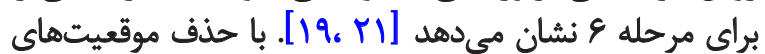

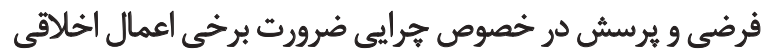

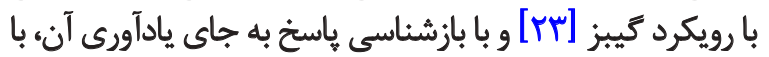

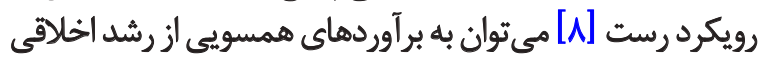

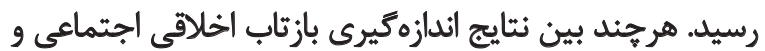

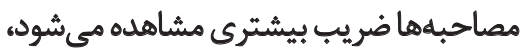

به نظر ميرسد اندازهكيرى بازتاب اخلاقى اجتماعى سطح باري

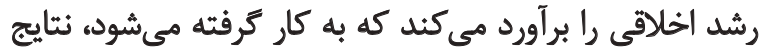


از فرايندهاي يردازش اطلاعات متفاوتى استفاده مي كثنده لذا

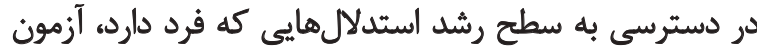

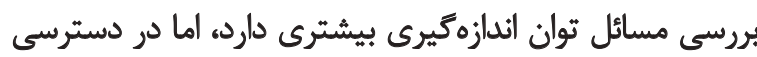

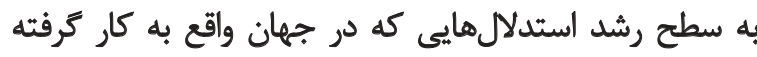
مىشود اندازمخيرى بازثاب اخلاقى اجتماعى نتايج بهترى به دانه دست مي دهن.

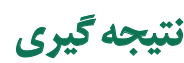

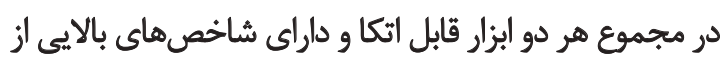

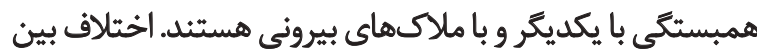

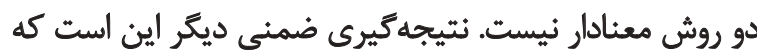

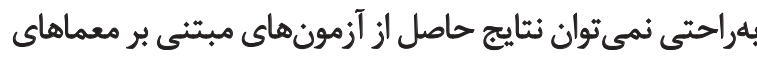

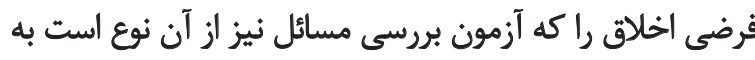
تصميمات جهان واقع تعميم داد.

خازجمله محدوديتهاي ئروهش حاضر، استفاده از نمونهاي

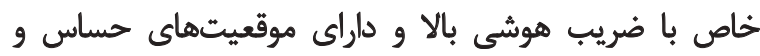

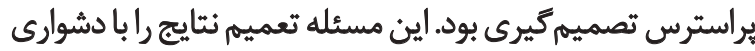

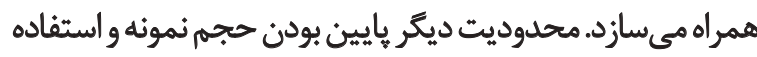
از روش نمونه كيرى هدفمند به جاى نمونه

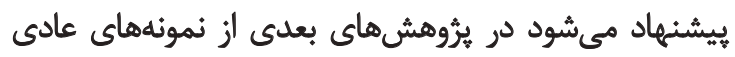

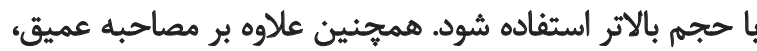

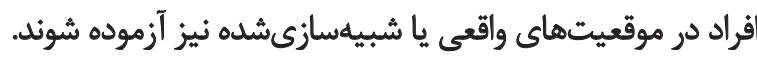

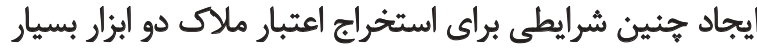
كمك كنينده خواهد بود.

$$
\text { سياسكزן إىى }
$$

مقاله حاضر بركرفته از رساله دكترى نويسنده اول در كروه

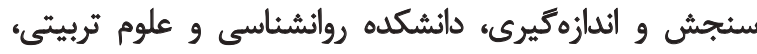
دانشكاه علامه طباطبايى تهران است. 


\section{References}

[1] Messick S. Meaning and values in test validation: The science and ethics of assessment. ETS Research Report Series. 1988; 1988(2):i-28. doi: 10.1002/j.2330-8516.1988.tb00303.x

[2] Haidt J. Morality. Perspectives on Psychological Science. 2008; 3(1):65-72. doi: 10.1111/j.1745-6916.2008.00063.x

[3] McFerran B, Aquino K, Duffy M. How Personality and Moral Identity Relate to Individuals' Ethical Ideology. Business Ethics Quarterly. 2010; 20(01):35-56. doi: 10.5840/beq20102014

[4] Christensen JF, Gomila A. Moral dilemmas in cognitive neuroscience of moral decision-making: A principled review. Neuroscience \& Biobehavioral Reviews. 2012;36(4):1249-64. doi: 10.1016/j. neubiorev.2012.02.008

[5] Pittel SM, Mendelsohn GA. Measurement of moral values: A review and critique. Psychological Bulletin. 1966; 66(1):22-35. doi: $10.1037 /$ h0023425

[6] Lind G. The cross-cultural validity of the Moral Judgment Test: Findings from 29 cross-cultural studies. Paper Presented at The Conference of the American Psychological Association; 2005; Massachusetts, USA.

[7] Gibbs JC. Moral development and reality: Beyond the theories of Kohlberg, Hoffman, and Haidt. Oxford: Oxford University Press; 2013. doi: 10.1093/acprof:osobl/9780199976171.001.0001

[8] Rest JR, editor. Moral development in the professions: Psychology and applied ethics. New York: Psychology Press; 1994.

[9] Haidt J, Graham J. When Morality Opposes Justice: Conservatives Have Moral Intuitions that Liberals may not Recognize. Social Justice Research. 2007; 20(1):98-116. doi: 10.1007/s11211007-0034-z

[10] Lapsley DK. Moral stage theory. In Killen M, Smetana J, editors. Handbook of moral development. New York: Psychology Press; Psychology Press; 2006.

[11] Colby A, Kohlberg L, Gibbs J, Lieberman M, Fischer K, Saltzstein HD. A longitudinal study of moral judgment. Monographs of the Society for Research in Child Development. 1983; 48(1-2):1. doi: 10.2307/1165935

[12] Kohlberg L. Stages of moral development. Moral Education. 1971;1:23-92.

[13] Boom J, Brugman D. Measuring moral development in adolescents. In: A. Wouter Haaften editor. Moral Sensibilities and Education; Volume 3: The adolescent. Dublin: Concorde Pub. 2005.

[14] Colby A, Kohlberg L. The measurement of moral judgement: Volume 2, Standard Issue Scoring Manual. Cambridge: Cambridge University Press; 1987.

[15] Boom J, Brugman D, van der Heijden PGM. Hierarchical Structure of Moral Stages Assessed by a Sorting Task. Child Development. 2001 Mar; 72(2):535-48. doi: 10.1111/1467-8624.00295

[16] Rest JR, Narvaez D, Thoma SJ, Bebeau MJ. DIT2: Devising and testing a revised instrument of moral judgment. Journal of Educational Psychology. 1999; 91(4):644-59. doi: 10.1037/00220663.91.4.644
[17] Kohlberg L, Hersh RH. Moral development: A review of the theory. Theory into Practice. 1977; 16(2):53-9. doi: $10.1080 / 00405847709542675$

[18] Thoma SJ, Dong Y. The Defining Issues Test of moral judgment development. Behavioral Development Bulletin. 2014; 19(3):5561. doi: $10.1037 / \mathrm{h} 0100590$

[19] Rest JR, Narvaez D, Thoma SJ, Bebeau MJ. A Neo-Kohlbergian Approach to Morality Research. Journal of Moral Education. 2000; 29(4):381-95. doi: 10.1080/713679390

[20] Narvaez D, Bock T. Moral Schemas and Tacit Judgement or How the Defining Issues Test is Supported by Cognitive Science. Journal of Moral Education. 2002; 31(3):297-314. doi: $10.1080 / 0305724022000008124$

[21] Rest J, Thoma SJ, Narvaez D, Bebeau MJ. Alchemy and beyond: Indexing the Defining Issues Test. Journal of Educational Psychology. 1997; 89(3):498-507. doi: 10.1037/0022-0663.89.3.498

[22] Bebeau MJ. The Defining Issues Test and the Four Component Model: Contributions to professional education. Journal of Moral Education. 2002; 31(3):271-95. doi: 10.1080/0305724022000008115

[23] Gibbs JC, Basinger KS, Fuller D, Fuller RL. Moral maturity: Measuring the development of sociomoral reflection. Abingdon: Routledge; 2013.

[24] Emler N, Tarry H, James AS. Post-conventional moral reasoning and reputation. Journal of Research in Personality. 2007; 41(1):76-89. doi: 10.1016/j.jp. 2006.02.003

[25] Loftus GR. Comparison of recognition and recall in a continuous memory task. Journal of Experimental Psychology. 1971; 91(2):220-6. doi: 10.1037/h0031841

[26] Xu Y, Iran-Nejad A, Thoma SJ. Administering defining issues test online: Do response modes matter. Journal of Interactive Online Learning. 2007; 6(1):10-27.

[27] Moon YL. A Review of Cross-Cultural Studies on Moral Judgment Development Using the Defining Issues Test. Behavior Science Research. 1986; 20(1-4):147-77. doi: $10.1177 / 106939718602000107$

[28] Page R, Bode J. Comparison of Measures of Moral Reasoning and Development of a New Objective Measure. Educational and Psychological Measurement. 1980; 40(2):317-29. doi: 10.1177/001316448004000206

[29] Davison ML, Robbins S. The Reliability and Validity of Objective Indices of Moral Development. Applied Psychological Measurement. 1978; 2(3):391-403. doi: 10.1177/014662167800200314

[30] Dawson TL. A comparison of three developmental stage scoring systems. Journal of applied measurement. 2002; 3(2):146-89. PMID: 12011499

[31] Gibbs JC, Basinger KS, Grime RL, Snarey JR. Moral judgment development across cultures: Revisiting Kohlberg's universality claims. Developmental Review. 2007; 27(4):443-500. doi: 10.1016/j.dr.2007.04.001

[32] Krebs DL, Denton K. Toward a more pragmatic approach to morality: A critical evaluation of Kohlberg's model. Psychological review. 2005; 112(3):629-49. doi: 10.1037/0033-295x.112.3.629

[33] Sharifi HP, Sharifi N. [Principles of Psychometrics and Psychosis (Persian)]. Tehran: Roshd Publications; 2015. 
[34] Creswell JW, Clark VL. Designing and conducting mixed methods research [AR. Kiamanesh, J. Saraei, Persian trans]. Tehran: Aish Publication; 2007.

[35] Delavar A. [Research methods in psychology (Persian)]. Tehran: Virayesh Publications; 2006

[36] Ghiasizadeh M. [Relationship between mental health and academic performance with moral judgment development in female secondary school students (Persian)]. Woman and Culture. 2012; 3(10):111-122.

[37] Kadivar P. [Moral psychology (Persian)]. Tehran: Agah Publication; 2004

[38] Delavar A., Minaei A, Eskandari H, Hooshyari Z. [Developed and standardize of the measurement tools for moral schemas and that's structural model based on personality factors, empathy and moral atmosphere [PhD Dissertation] (Persian)]. Tehran: Allameh Tabataba'i University; 2017.

[39] Braun V, Clarke V. Using thematic analysis in psychology. Qualitative Research in Psychology. 2006; 3(2):77-101. doi: 10.1191/1478088706qp063oa

[40] Hooshyari Z, Delavar A, Minaei A, Eskandari H. [Extraction of moral schemas in managers in face of moral dilemma (Persian)]. Ethics in science and Technology. 2017; 12 (3) :25-33.

[41] Boyce C, Neale P. Conducting in-depth interviews: A guide for designing and conducting in-depth interviews for evaluation input. Watertown: Pathfinder International; 2006.

[42] Eisenberg N. Empathy-related responding: Links with self-regulation, moral judgment, and moral behavior. In M. Mikulincer, P. R. Shaver editors. Prosocial Motives, Emotions, and Behavior: The Better Angels of Our Nature. Washington, D. C.: American Psychological Association; 2010. doi: 10.1037/12061-007

[43] Berenguer J. The Effect of Empathy in Environmental Moral Reasoning. Environment and Behavior. 2008; 42(1):110-34. doi: $10.1177 / 0013916508325892$

[44] Bretz S, Sun R. Two Models of Moral Judgment. Cognitive Science. 2017; doi: 10.1111/cogs.12517

[45] Aquino K, Freeman D, Reed A, Lim VKG, Felps W. Testing a social-cognitive model of moral behavior: The interactive influence of situations and moral identity centrality. Journal of Personality and Social Psychology. 2009; 97(1):123-41. doi: 10.1037/ a0015406

[46] Høst K, Brugman D, Tavecchio L, Beem L. Students' Perception of the Moral Atmosphere in Secondary School and the Relationship Between Moral Competence and Moral Atmosphere. Journal of Moral Education. 1998; 27(1):47-70. doi: 10.1080/0305724980270104

[47] Reynolds SJ, Ceranic TL. The effects of moral judgment and moral identity on moral behavior: An empirical examination of the moral individual. Journal of Applied Psychology. 2007; 92(6):1610-24. doi: 10.1037/0021-9010.92.6.1610

[48] Bartels DM, Bauman CW, Cushman FA, Pizarro DA, McGraw AP. Moral Judgment and Decision Making. The Wiley Blackwell Handbook of Judgment and Decision Making . 2015; 478-515. doi: 10.1002/9781118468333.ch17

[49] Francis KB, Howard C, Howard IS, Gummerum M, Ganis G, Anderson G, et al. Correction: Virtual Morality: Transition- ing from Moral Judgment to Moral Action? Plos One. 2017 12(1):e0170133. doi: 10.1371/journal.pone.0170133 
